\title{
Low levels of transposable element activity in \\ Drosophila mauritiana: causes and consequences
}

\author{
Robert Kofler and Christian Schlötterer*
}

April 20, 2015

\begin{abstract}
Transposable elements (TEs) are major drivers of genomic and phenotypic evolution, yet many questions about their biology remain poorly understood. Here, we compare TE abundance between populations of the two sister species D. mauritiana und D. simulans and relate it to the more distantly related $D$. melanogaster. The low population frequency of most TE insertions in D. melanogaster and D. simulans has been a key feature of several models of TE evolution. In D. mauritiana, however, the majority of TE insertions are fixed $(66 \%)$. We attribute this to a lower transposition activity of up to $47 \mathrm{TE}$ families in D. mauritiana, rather than stronger purifying selection. Only three families, including the extensively studied Mariner, may have a higher activity in D. mauritiana. This remarkable difference in TE activity between two recently diverged Drosophila species $(\approx 250,000$ years), also supports the hypothesis that TE copy numbers in Drosophila may not reflect a stable equilibrium where the rate of $\mathrm{TE}$ gains equals the rate of $\mathrm{TE}$ losses by negative selection. We propose that the transposition rate heterogeneity results from the contrasting ecology of the two species: the extent of vertical extinction of TE families and horizontal

\footnotetext{
*corresponding author: schlotc@gmail.com
} 
acquisition of active TE copies may be very different between the colonizing D. simulans and the island endemic D. mauritiana. Our findings provide novel insights in the evolution of TEs in Drosophila and suggest that the ecology of the host species could be a major, yet underappreciated, factor governing the evolutionary dynamics of TEs.

\section{Significance statement}

Transposable elements (TE) are stretches of DNA that selfishly propagate within genomes. By comparing TE abundance between closely related Drosophila species we found that TE activity is significantly lower in an island endemic species than in worldwide distributed species. We propose that geographic isolation on islands could lead to fewer opportunities for acquiring active TEs by horizontal transfer as well as an accelerated loss of functional TEs. Our results suggest that ecology of host species may be a powerful factor which modulates the activity of multiple TE families even over very short evolutionary time scales.

\section{Introduction}

Transposable elements (TEs) are stretches of DNA that selfishly spread within genomes, even to the detriment of the host (Hickey, 1982). Insertions of TEs in host genomes may have a significant impact on phenotypes, including diverse phenomena such as variation of quantitative traits (Mackay et al., 1992), human diseases (Kazazian Jr, 1998), environmental adaptation (Casacuberta and González, 2013) and genome evolution (Kazazian, 2004). The evolutionary dynamics of TEs have been extensively studied, especially in the model organism D. melanogaster (Burt and Trivers, 2008). One particularly interesting feature that has emerged from these studies is that the vast majority of TE insertions in D. melanogaster tend to be at low population frequencies (Charlesworth et al., 1994, 1992; Sniegowski and Charlesworth, 1994; Biémont et al., 1994; Petrov et al., 2011; Montgomery and Langley, 1983; Kofler et al., 2012; Brookfield, 1986; Maumus et al., 2015). Recently, this pattern was also found in the closely related D. simulans (Kofler et al., 2014). Fixed TE insertions are 
largely restricted to low recombining regions (Bartolomé and Maside, 2004; Bartolomé et al., 2002; Kofler et al., 2012; Petrov et al., 2011) and to a few TE families (Kofler et al., 2012; Petrov et al., 2011, 2003; Hey, 1989). Two competing, but not mutually exclusive, models have been proposed to account for this predominance of low frequency insertions (Barrón et al., 2014). The transposition-selection balance model states that the abundance of most TE families is in an equilibrium, where the gain of novel insertions due to transposition equals the loss of copies by negative selection. (Charlesworth and Langley, 1989; Petrov et al., 2003, 2011; Nuzhdin, 1999; Blumenstiel et al., 2013) . According to this model the low population frequency of TE insertions is mostly due to strong purifying selection acting against TE insertions. By contrast, the transposition burst model assumes that TE insertions with low frequencies are the consequences of recent bursts of TE activity (Bergman and Bensasson, 2007; Lerat et al., 2011; Kofler et al., 2012).

A recent comparison of $D$. simulans and D. melanogaster identified substantial differences in TE abundance between these two species (Kofler et al., 2014). We proposed that these differences were probably due to an increased TE activity that could have been triggered by the recent habitat expansion of the two species (Kofler et al., 2014). If this hypothesis holds, endemic species are expected to have lower TE activities and thus fewer TE insertions with low frequencies.

We show that in contrast to D. melanogaster and D. simulans most TE insertions in the island endemic D. mauritiana are fixed (66\%). We propose that differences in the abundance of low frequency insertions between $D$. mauritiana $(f \leq 0.2,18.0 \%)$ and $D$. simulans $(64.3 \%)$, are likely due to different activities of up to $47 \mathrm{TE}$ families. This suggests that activity of multiple TE families in Drosophila substantially changed over very short evolutionary time scales (<250,000 years), lending support to the transposition burst model of TE evolution. We propose that these differences in TE activity could be due to the different ecologies of the two species which may result in different opportunities for acquiring active TEs by horizontal transfer and different rates of loss of active TE families by vertical extinction. 


\section{Results}

Short read sequencing from pooled individuals [Pool-seq (Schlötterer et al., 2014)] has been shown to be an excellent approach to measure the population frequency of TE insertions on the genomic scale (Kofler et al., 2012, 2014; Kim et al., 2014). We compared TE abundance in a population of the island endemic D. mauritiana [data from Nolte et al. (2012)] to populations of the two cosmopolitan species D. simulans and D. melanogaster [data from Kofler et al. (2014); Nolte et al. (2012)]. The D. simulans and D. melanogaster populations were sampled in 2013 in Kanonkop (South Africa) (Kofler et al., 2014) and D. mauritiana was sampled between 2006 and 2009 from multiple locations in Mauritius (Nolte et al., 2012). We annotated TE insertions in all three reference genomes de novo (supplementary material and methods 4.2) as outlined in Kofler et al. (2014) and created a TE annotation for the $D$. mauritiana reference genome (Nolte et al., 2012). The TE abundance was estimated with PoPoolationTE (Kofler et al., 2012) after standardizing the physical coverage [numbers of paired-end reads spanning a TE insertion site (Meyerson et al., 2010)] to 60 in all populations. We only considered TE insertions in orthologous regions, i.e. regions present in the assemblies of all three species (see supplementary material and methods 4.2). This procedure permits a direct comparison of TE abundance between species. The impact of the various steps in our pipeline is detailed for every TE family in supplementary file 2 .

\section{TE abundance in D. mauritiana and D. simulans}

D. mauritiana contains significantly fewer TE insertions than D. simulans (Dmau $=2,764$, $D \operatorname{sim}=8,056$; Chi-square test, $\left.\chi^{2}=2,588.3, p<2.2 e-16\right)$ and this pattern is seen for all three TE orders $($ LTR Dmau $=532$, Dsim $=1,811$; non-LTR Dmau $=404$, Dsim = 1, 259; TIR Dmau $=1,787, D \operatorname{sim}=4,737)$. Out of 7,097 D. simulans insertions for which population frequency estimates could be obtained (non-overlapping TE insertions) 1,516 $(21.4 \%)$ are fixed ( $f \geq 0.9$; allowing for some error) while in D. mauritiana, 1,710 out of 2, $586(66.1 \%)$ insertions are fixed (supplementary table 1). Despite the lower number of TE insertions D. mauritiana has more fixed insertions than D. simulans (Dmau $=1,710$, 
$D \operatorname{sim}=1,516$, Chi-square test, $\left.\chi^{2}=11.7, p=0.00063\right)$. This difference in fixed TE insertions is largely explained by a few TE families $(f \geq 0.9$; top three in descending order INE-1: Dmau =1,140, Dsim = 996; roo: Dmau =88, Dsim =67; Cr1a: Dmau = 43, Dsim = 32). The striking difference in overall copy numbers between the two species is mostly due to the about tenfold higher abundance of low frequency insertions in D. simulans $\left(f \leq 0.2 ;\right.$ Dmau $=466(18.0 \%), D \operatorname{sim}=4,562(64.3 \%) ; \chi^{2}=3,336.8, p<2.2 e-16 ;$ supplementary fig. 1). This difference in low frequency insertions holds for all chromosome arms (fig. 1; supplementary table 1) and all TE orders $(f \leq 0.2$; TIR Dsim $=2,629$, Dmau $=191$, Chi-square test, $\chi^{2}=2,107.7, p<2.2 e-16 ;$ LTR Dsim $=1,147$, Dmau $=$ 153, Chi-square test, $\chi^{2}=760.0, p<2.2 e-16$; non-LTR Dsim $=746$, Dmau $=129$, Chisquare test, $\left.\chi^{2}=435.1, p<2.2 e-16\right)$. A more detailed analysis showed that $47 \mathrm{TE}$ families had significantly fewer low frequency insertions in D. mauritiana $(f \leq 0.2$; Chi-square test $p \leq 0.05$; fig. 2), while only 3 families, including the intensely studied Mariner (Hartl et al., 1997; Lohe et al., 1995; Jacobson et al., 1986), had fewer low frequency insertions in $D$. simulans (fig. 2).

\section{Robustness of the contrasting TE abundance pattern}

Given the implicit challenges of cross-species comparisons, we carefully scrutinized our analysis to ensure that our result reflect a biological pattern rather than an artefact of the analysis: 1) Since the reported difference in low frequency insertions is consistently found in all steps of our pipeline (supplementary file 2) we can rule out that one or more filtering steps in the pipeline have caused this pattern. Even in the least processed data, $7.3 \%$ of the reads align to TE sequences in D. simulans while only $4.3 \%$ align to TEs in D. mauritiana (supplementary file 2). 2) Even without adjusting the physical coverage in both species, we find fewer TE insertions in D. mauritiana, despite this data set has a higher coverage (supplementary file 2; supplementary material and methods 4.1). 3) The D. simulans and D. melanogaster data were obtained from a single population but the $D$. mauritiana sample was composed of flies from multiple collections at different time points and locations (Nolte 
et al., 2012; Kofler et al., 2014). Although no population structure could be detected in $D$. mauritiana (Nunes et al., 2010), we tested if combining samples from different populations may cause a bias against low frequency TE insertions. We used an additional D. simulans population composed of flies sampled from multiple locations at different years (central Africa between 2001 and 2009; for an overview of all population see supplementary table 3). Although this D. simulans population has markedly fewer reads than the D. mauritiana population, which strongly favours identification of low frequency insertions in D. mauritiana, we still found a highly significant excess of low frequency insertions in D. simulans $\left(f \leq 0.2 ;\right.$ Dsim $_{c a}=2,911$, Dmau $=466, \chi^{2}=1770.2 p<2.2 e-16 ;$ supplementary file 2). 4.) It may be possible that a higher sequence divergence of TE insertions in D. mauritiana results in a smaller fraction of mapped TE reads and thus a lower abundance of TE insertions. Nevertheless, we consider this hypothesis unlikely since our pipeline takes sequence divergence into account by mapping reads to consensus TE sequences as well as to all diverged copies of a TE family found in a reference genome (using RepeatMasker and sensitive search settings). Furthermore, the same de novo annotation procedure, which relies on consensus TE sequences mostly derived from D. melanogaster (Quesneville et al., 2005), has been applied to both species. Since both species split after the divergence from D. melanogaster they are expected to have a similar divergence to D. melanogaster (Nolte et al., 2012). In agreement with this we detect no lineage specific TE families, i.e. we find more than 100 reads mapping to all TE families in both species [with two exceptions: the P-element is missing in D. mauritiana (Kofler et al., 2015) and Stalker3 is absent in D. simulans; supplementary file 2]. Additionally, sequence divergence is expected to affect fixed TE insertions, which are enriched for old and inactive TE families (Kofler et al., 2014, 2012), more than low frequency insertions which are mostly young insertions derived from active copies that preserved functionality by escaping accumulation of mutations. In contrast to this expectation we found significantly more fixed TE insertions in D. mauritiana than in D. simulans, which suggests that higher sequence divergence of D. mauritiana TEs is not affecting our results. 5.) In contrast to the trend of a higher TE abundance in D. simulans 
in our data, Mariner insertions were previously shown to be more abundant in D. mauritiana (Jacobson et al., 1986; Hartl et al., 1997). Since our analyses confirm this pattern $(\operatorname{Dsim}=4, D m a u=11$; supplementary file 2), we conclude that our observation of a low TE abundance in D. mauritiana is not due to a general bias against identification of TE insertions in this species.

\section{Causes for the contrasting TE abundance pattern}

Which evolutionary forces could be responsible for the parallel divergence of the number of low-frequency insertions across multiple TE families between two closely related species? In the following we discuss two, not mutually exclusive, hypotheses: 1) differential TE activity and 2) different selection efficacy. Since most TE insertions are deleterious (Burt and Trivers, 2008), differences in selection efficacy between species could cause the observed pattern. Both the population size (Charlesworth and Charlesworth, 1983; Kofler et al., 2014; Gonzalez and Petrov, 2012) and the recombination rate (Dolgin and Charlesworth, 2008; Kofler et al., 2012) have frequently been shown to affect the efficacy of selection against TE insertions in natural populations. Since the efficacy of selection is higher in large populations (Hartl and Clark, 1997), the number of TE insertions, including low frequency insertions, should be lower in large populations (Kofler et al., 2014; Gonzalez and Petrov, 2012). We used the nucleotide diversity $\pi$ (Nei and Li, 1979) to compare the population size estimates in both species. The nucleotide diversity in D. mauritiana is lower than in D. simulans (average over all 100kb windows in orthologous regions; $\pi_{\text {Dmau }}=0.0085, \pi_{\text {Dsim }}=0.0112 ;$ fig. 1 ) suggesting that $D$. mauritiana has a smaller population size than D. simulans, which is also consistent with the geographic distribution of the two species (Lachaise et al., 1988). However, a smaller population size as found in D. mauritiana could also lead to a loss of low frequency insertions by decreasing the efficacy of selection, thus allowing TEs to more rapidly fix (Lee and Langley, 2010). We consider it unlikely that this could be responsible for a reduced abundance of low frequency insertions in $D$. mauritiana: out of the $47 \mathrm{TE}$ families with significantly fewer low frequency insertions in D. mauritiana, only 24 (51\%) have more 
fixed insertions in D. mauritiana while the remaining 23 families (49\%) either have equal or higher numbers of fixed insertions in D. simulans. Consequently it is unlikely that differences in the population size between the two species could account for the divergent abundance of low frequency insertions.

Alternatively a higher recombination rate could result in more ectopic recombination and less linkage between sites and therefore to an increased selection intensity against TE insertions (Dolgin and Charlesworth, 2008; Kofler et al., 2012). The genetic map of D. mauritiana is about 1.4 times $(=1.8 / 1.3)$ larger than the map of D. simulans (True et al., 1996). Assuming equal genome sizes for both species (Boulesteix et al., 2006), D. mauritiana should have an about 1.4 times higher recombination rate than D. simulans, suggesting that purifying selection against TE insertions is stronger in D. mauritiana. To test if recombination rate were influencing the abundance of low frequency insertions we mad use of recombination rate differences among chromosomes. True et al. (1996) reported that D. mauritiana chromosomes X, 2 and 3 have an about $1.8(=1.8 / 1.0), 1.23(=1.6 / 1.3)$ and $1.235(=2.1 / 1.7)$ fold larger genetic map than D. simulans chromosomes. Thus the X chromosome has the most pronounced differences in recombination rate between the two species and chromosome 2 the least. Despite these differences in recombination rates both chromosomes showed similar heterogeneity in the number of low frequency insertions between the two species $\left(f \leq 0.2, \operatorname{Dmau}_{X}=104, \operatorname{Dsim}_{X}=935, \operatorname{Dmau}_{2}=174, D_{\operatorname{sim}}=1,652\right.$, Fishers exact test, $p=0.6938)$. Since, $\mathrm{X}$-chromosome and autosomes differ in many features other than recombination rates (Vicoso and Charlesworth, 2006), we further exploited local recombination rate heterogeneity on chromosome 3L (True et al., 1996). While the chromosome-wide recombination rate is higher in $D$. mauritiana, the local recombination rate between polytene bands 250 and 500 (measured from the centromere) on chromosome 3L is higher in $D$. simulans (True et al., 1996). Nevertheless, we still find fewer low frequency TE insertions in this genomic region in D. mauritiana (Chi-square test; $p<2.2 e-16$; supplementary results 3.1). This suggests that recombination rate differences are not sufficient to explain the differences in TE composition between D. simulans and D. mauritiana. 
Irrespective of whether selection efficacy is mediated by recombination rate or effective population size, differences will be reflected in the site frequency spectrum: stronger purifying selection results in a lower frequency of segregating TEs. To avoid misleading signals from ancestral insertions that occurred before the two species split, we only focussed on species specific TE insertions and compared the mean population frequencies. Interestingly, we found a higher mean population frequency of lineage specific TEs in D. mauritiana than in D. simulans $($ Dmau $=0.493$, Dsim $=0.147$, Wilcox rank sum test $W=3391131, p<$ $2.2 e-16)$. The higher population frequency of $D$. mauritiana insertions is consistent for all three TE orders (LTR Dmau $=0.419$, Dsim $=0.153$, Wilcox rank sum test $W=226682.5$, $p<2.2 e-16$; non-LTR Dmau $=0.341$, Dsim $=0.156$, Wilcox rank sum test $W=101537$, $p=2.891 e-06 ;$ TIR Dmau $=0.606, D$ sim $=0.144$, Wilcox rank sum test $W=981319$, $p<2.2 e-16$; TIR without INE-1 Dmau $=0.264$, Dsim $=0.106$, Wilcox rank sum test $W=300776, p=0.0042$ ). Furthermore, 29 out of 39 TE families, with significantly different abundance of low frequency insertions between both species (fig. 1) and at least one lineage specific TE insertion in both species (39 out of 47), had on average a higher population frequency in D. mauritiana while 10 had a higher population frequency in $D$. simulans (supplementary file 3 ). The elevated population frequencies of D. mauritiana specific TE insertions persist when we exclude fixed insertions $f \geq 0.9 ;$ Dmau $=0.262$, Dsim $=0.117$, Wilcox rank sum test $W=1860001, p<5.1 e-15)$.

Given that a range of different tests failed to provide convincing support for the hypothesis that the efficacy of selection against TE insertions explains the lower number of segregating TEs in D. mauritiana compared to D. simulans, an alternative explanation is required. We propose that the transposition activity differs between the two species, with the majority of families being more active in D. simulans (47 families). Nevertheless, $3 \mathrm{TE}$ families, including the well-studied Mariner element (Hartl et al., 1997; Lohe et al., 1995; Jacobson et al., 1986), have more low frequency insertions in D. mauritiana and may be more active in this species (supplementary file 4). 


\section{Comparison with D. melanogaster}

It may be tempting to assume that low TE activity in D. mauritiana is a derived property, as $D$. melanogaster and $D$. simulans both have more low frequency insertions $(f \leq 0.2$; Dmau $=466(18.0 \%)$, Dmel $=9,488$ (81.7\%), Dsim $=4,562$ (64.3\%), supplementary file 4) and consequently may have more active TEs. We caution, however, that this interpretation is too simplistic, since the rapid activity change between $D$. mauritiana and $D$. simulans suggests that several changes in TE activity may have occurred since the split of D. melanogaster and the D. simulans group. Despite D. simulans and D. melanogaster sharing a high TE activity, the profile of active TE families in D. simulans is more similar to D. mauritiana than to D. melanogaster (Spearman correlation of the abundance of low frequency insertions, $f<0.2$, for all TE families; Dmau-Dsim $\rho=0.58, p=8.3 e-11$; Dsim-Dmel $\rho=0.43, p=4.9 e-06$; supplementary file 4).

\section{Discussion}

In this report we compare for the first time the genomic distribution of TE insertions in three closely related Drosophila species on a population scale. By standardizing the Pool-Seq data to the same physical coverage and using an identical pipeline for TE identification in all three species we minimize potential biases of interspecific comparisons. While the TE landscape of D. simulans and D. melanogaster populations fit the previously described predominance of low frequency TE insertions (Charlesworth et al., 1994, 1992; Sniegowski and Charlesworth, 1994; Biémont et al., 1994; Petrov et al., 2011; Montgomery and Langley, 1983; Kofler et al., 2012 , 2014), in D. mauritiana, the pattern is fundamentally different. We show that the island endemic D. mauritiana not only has fewer TE insertions than the other two species, but the insertions have a significantly higher population frequency, with the majority of them being fixed. This unexpected TE distribution could be explained either by stronger purifying selection in D. mauritiana, removing novel insertions, or a higher transposition rate in D. simulans and D. melanogaster. We carefully scrutinized the D. mauritiana data 
for any signals of higher selection efficacy, but did not detect support for this hypothesis. Therefore, we concluded that transposition rate heterogeneity is the most likely explanation for the contrasting TE distribution between the species. Such rapid changes in TE activity affecting a broad range of TE families has only previously been reported in plants. One particular impressive example is the explosive activity of 11 LTR families in maize which led to a doubling of the genome size within 3 million years (SanMiguel et al., 1998). No marked differences in genome size were, however, reported for D. simulans and D. mauritiana (Boulesteix et al., 2006), probably because the higher TE activity in D. simulans is mostly reflected in a high abundance of low frequency insertions, which have on the average a small impact on genome size. We do not consider it very likely that a similar genome expansion will be seen in $D$. simulans since the large effective population size allows for a very efficient selection against deleterious TE insertions.

With the three Drosophila species being closely related and sharing almost all TE families, it is very unlikely that simple structural differences could be responsible for the divergence in transposition rates. Rather, we propose that two different, but not mutually exclusive, processes related to the contrasting ecology of the species may be responsible for the heterogeneity in transposition rates. First, environmental stress may activate TEs (Capy et al., 2000) and colonizing species, like D. simulans and D. melanogaster (Lachaise et al., 1988), may be exposed to more environmental stress than species that remained in the ancestral habitat such as D. mauritiana (Lachaise et al., 1988). So far, stress was only shown to activate a few TE families, like 412 and hobo (Capy et al., 2000), and therefore it remains unclear if stress can account for the observed activation of $47 \mathrm{TE}$ families. Second, the balance between the two opposing forces of vertical extinction and horizontal transmission may be shifted between D. simulans and D. mauritiana. Vertical extinction, i.e. the loss of active TE copies, may result from competition between TE families, the accumulation of deleterious mutations and the evolution of host repression of TEs (Burt and Trivers, 2008). On the other hand, active TE copies may be gained by horizontal transmission from different species (Bartolomé et al., 2009; Sánchez-Gracia et al., 2005; Silva et al., 2004). The num- 
ber of active TE copies segregating in a population may thus be the outcome of these two opposing forces. Using a simple model Kaplan et al. (1985) showed that vertical extinction may be rapid in small populations, and we found that D. mauritiana likely has a smaller population size than D. simulans. Furthermore, the colonizing D. simulans may have had more opportunities for acquiring active TEs by HT than the island endemic D. mauritiana, especially given that opportunities for HT increase with population size and species diversity in the habitat, both of which may be low for island endemic species (MacArthur and Wilson, 1967). Reduced opportunity for HT has also been suggested as explanation for the low TE content of the island endemic D. grimshawi (Drosophila 12 Genomes Consortium, 2007) and could also be responsible for the high fraction of fixed insertions in the endemic D. algonquin (Hey, 1989). It is therefore possible that vertical extinction of TE families predominates in D. mauritiana while horizontal acquisition of active TE copies is more frequent in D. simulans. The presence of all TE families (except P-element and Stalker3) in the three species may be interpreted to counter this hypothesis, which requires some horizontal transfer of active TEs, as HT is expected to cause a patchy distribution of TEs in the phylogeny of species (Schaack et al., 2010; Loreto et al., 2008; Silva et al., 2004). However, abundant HT, as for example found for the P-element which invaded two Drosophila species within one century (Kofler et al., 2015), could also lead to the presence of all TE families in the three species.

Our results also have bearing on a long-standing debate about the evolutionary dynamics of TEs (Barrón et al., 2014). The transposition-selection balance model assumes that the abundance of most TE families reflects the equilibrium of gains of novel insertions by transpositions and the loss of copies by negative selection. (Charlesworth and Langley, 1989; Petrov et al., 2003, 2011; Nuzhdin, 1999). Thus low population frequencies of TE insertions are the outcome of strong purifying selection acting against TE insertions. By contrast, according to the transposition burst model, TE insertions with low frequencies are the consequence of recent increase in TE activity (Kofler et al., 2012; Bergman and Bensasson, 2007; Lerat et al., 2011; Blumenstiel et al., 2013). Our finding of substantial differences in activity 
of multiple TE families between two closely related species at short time scales raises the important questions of whether the TE distribution in D. simulans and D. melanogaster has already reached an equilibrium state. Assuming that habitat expansions and stressful environments modulate TE activity, it appears possible that the distribution of TEs rarely reaches an equilibrium state. We anticipate that future work analyzing multiple populations of related species with different ecologies may shed further light on the forces shaping the evolutionary dynamics of TEs.

\section{Material and Methods}

We measured TE abundance in one population of $D$. mauritiana, two populations of $D$. simulans and one population of D. melanogaster using previously published Pool-seq data (Nolte et al., 2012; Kofler et al., 2014). For details about the samples used in this study see supplementary table 4.1 . We de novo annotated TE insertions in the reference genomes of D. simulans (r1.0 Palmieri et al., 2014), D. mauritiana (r1.0 Nolte et al., 2012) and D. melanogaster (v6.03; dos Santos et al., 2015) and identified TE insertions with PoPoolationTE (Kofler et al., 2012). In contrast to our previous work (Kofler et al., 2014) we included the canonical sequence of Mariner, which was discovered in D. mauritiana (Hartl et al., 1997), into our pipeline for estimating TE abundance. Pairwise nucleotide diversity was estimated for a natural population of D. mauritiana (Nolte et al., 2012) and a natural population of D. simulans from South Africa (Kofler et al., 2014) using PoPoolation (Kofler et al., 2011). Orthologous regions between D. simulans, D. melanogaster and D. mauritiana, i.e. regions occurring in the assemblies of all three species, were identified with MUMmer (v3.23; nucmer) (Kurtz et al., 2004). TE insertions at similar genomic positions in D. mauritiana and D. simulans were identified by reciprocally aligning $1000 \mathrm{bp}$ regions flanking each TE insertion to the respective reference genomes with bwa (v0.7.5a) (Li and Durbin, 2010) and scanning for insertions of the same family within these boundaries. All statistical analysis was done with R ( R Core Team, 2012). For details see supplementary material and methods. The TE annotation of the D. mauritiana genome 
and the TE abundance in the $D$. mauritiana population have been made publicly available (https://sourceforge.net/p/popoolationte/wiki/pdmau/).

\section{Acknowledgments}

We thank all members of the Institute of Population Genetics for feedback and support.

This work was supported by the ERC grant Archadapt. 


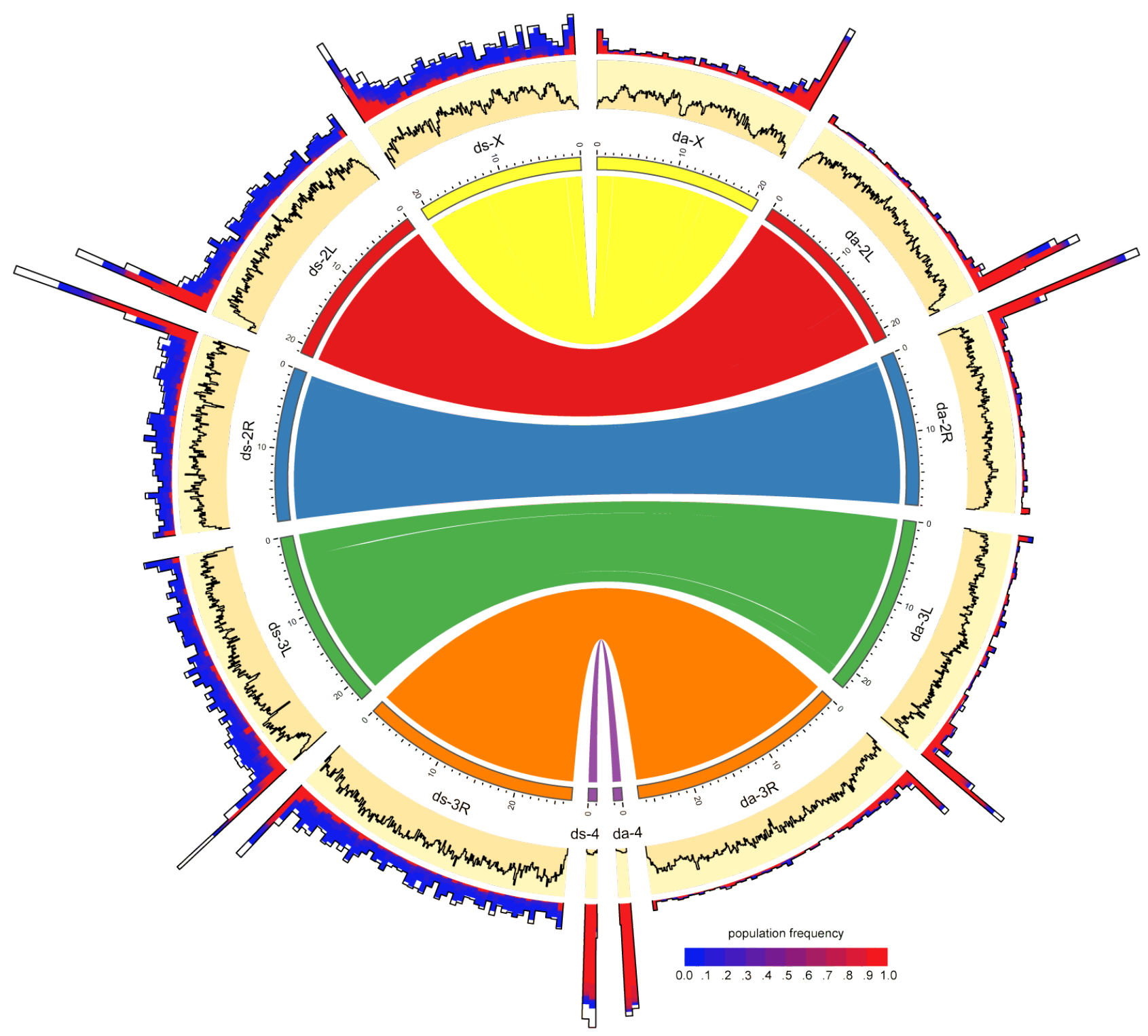

Figure 1: Distribution of TE insertions in a natural population of $D$. simulans (ds) and D. mauritiana (da). The TE distribution (outer graph) and the nucleotide polymorphism $\left(\Theta_{\pi}\right.$, yellow inner graph) is shown. TE abundance is shown for 500kb windows, whereas the nucleotide diversity is shown for 100kb windows. For overlapping TE insertions (white) no estimates of population frequencies could be obtained. The relationship between the reference genomes is shown in the inside. The maximum nucleotide diversity of the plot is 0.0192 and the maximum number of TE insertions 275 
bioRxiv preprint doi: https://doi.org/10.1101/018218; this version posted April 20, 2015. The copyright holder for this preprint (which was not
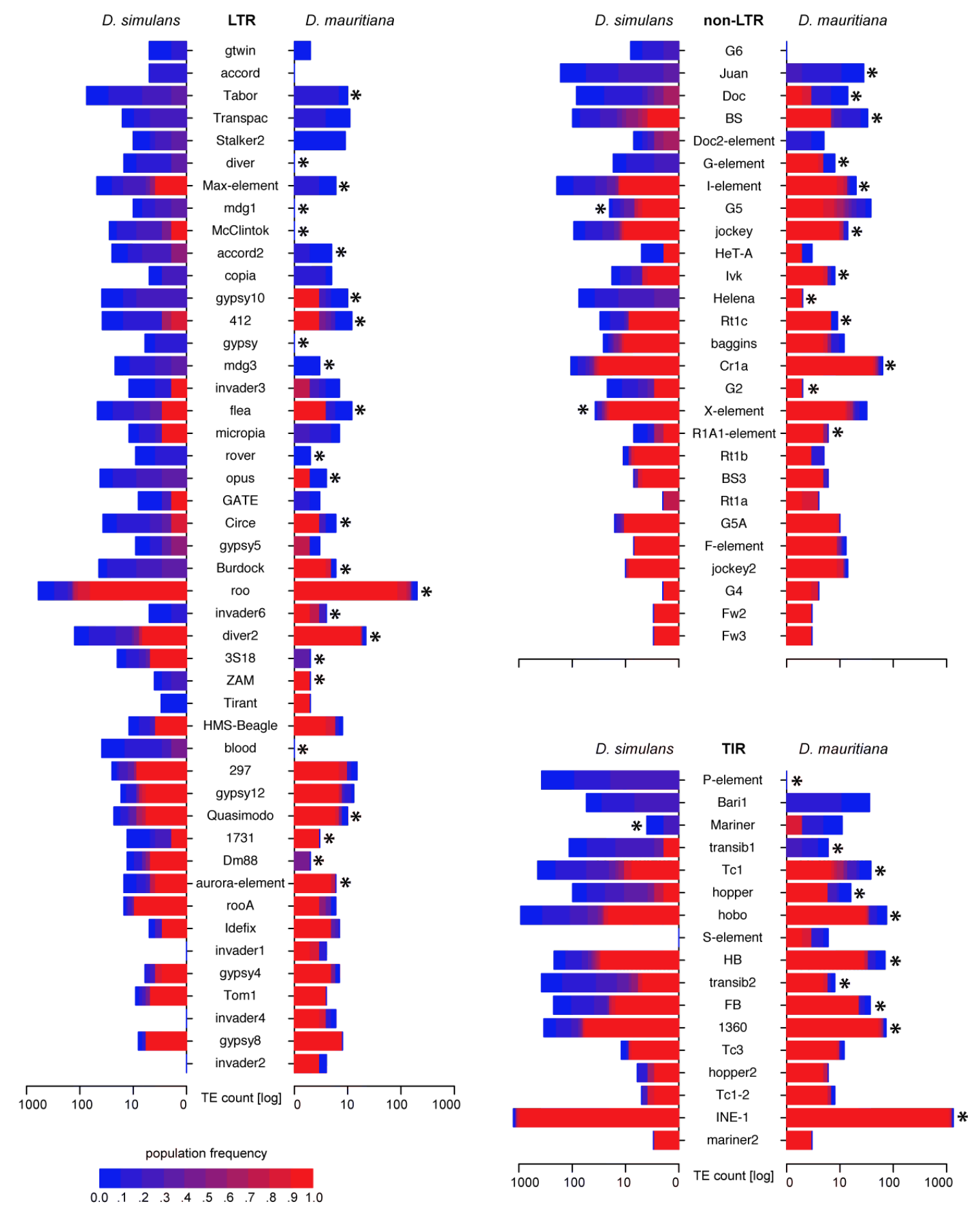

Figure 2: Abundance of different TE families in a natural D. simulans and D. mauritiana population. Only TE families with more than 10 insertions are shown. Significant differences in the abundance of low frequency insertions are indicated at the species having the lower counts ('*'; Chi-square test; $p<0.05)$. Follback is grouped with TIR solely for graphic reasons. 


\section{References}

Barrón, M. G., Fiston-Lavier, A.-S., Petrov, D. A., and González, J. (2014). Population genomics of transposable elements in drosophila. Annual Review of Genetics, 48(1).

Bartolomé, C., Bello, X., and Maside, X. (2009). Widespread evidence for horizontal transfer of transposable elements across Drosophila genomes. Genome biology, 10(2):R22.

Bartolomé, C. and Maside, X. (2004). The lack of recombination drives the fixation of transposable elements on the fourth chromosome of drosophila melanogaster. Genetical research, 83(02):91-100.

Bartolomé, C., Maside, X., and Charlesworth, B. (2002). On the abundance and distribution of transposable elements in the genome of drosophila melanogaster. Molecular biology and evolution, 19(6):926-937.

Bergman, C. M. and Bensasson, D. (2007). Recent LTR retrotransposon insertion contrasts with waves of non-LTR insertion since speciation in Drosophila melanogaster. Proceedings of the National Academy of Sciences of the United States of America, 104(27):11340-5.

Biémont, C., Lemeunier, F., Guerreiro, M., Brookfield, J., Gautier, C., Aulard, S., and Pasyukova, E. (1994). Population dynamics of the copia, mdg1, mdg3, gypsy, and p transposable elements in a natural population of drosophila melanogaster. Genetical research, 63(03):197-212.

Blumenstiel, J. P., Chen, X., He, M., and Bergman, C. M. (2013). An Age-of-Allele Test of Neutrality for Transposable Element Insertions. Genetics.

Boulesteix, M., Weiss, M., and Biémont, C. (2006). Differences in genome size between closely related species: the drosophila melanogaster species subgroup. Molecular biology and evolution, 23(1):162-167.

Brookfield, J. (1986). The population biology of transposable elements. Philosophical Transactions of the Royal Society B: Biological Sciences, 312(1154):217-226. 
Burt, A. and Trivers, R. (2008). Genes in conflict: the biology of selfish genetic elements. Belknap Press.

Capy, P., Gasperi, G., Biémont, C., and Bazin, C. (2000). Stress and transposable elements: co-evolution or useful parasites? Heredity, 85(2):101-106.

Casacuberta, E. and González, J. (2013). The impact of transposable elements in environmental adaptation. Molecular ecology, 22(6):1503-17.

Charlesworth, B. and Charlesworth, D. (1983). The population dynamics of transposable elements. Genetical Research, 42(01):1-27.

Charlesworth, B. and Langley, C. H. (1989). The population genetics of Drosophila transposable elements. Annual review of genetics, 23:251-87.

Charlesworth, B., Lapid, A., et al. (1992). The distribution of transposable elements within and between chromosomes in a population of drosophila melanogaster. i. element frequencies and distribution. Genetical research, 60(02):103-114.

Charlesworth, B., Sniegowski, P., and Stephan, W. (1994). The evolutionary dynamics of repetitive dna in eukaryotes.

Dolgin, E. S. and Charlesworth, B. (2008). The effects of recombination rate on the distribution and abundance of transposable elements. Genetics, 178(4):2169-2177.

dos Santos, G., Schroeder, A. J., Goodman, J. L., Strelets, V. B., Crosby, M. A., Thurmond, J., Emmert, D. B., Gelbart, W. M., et al. (2015). Flybase: introduction of the drosophila melanogaster release 6 reference genome assembly and large-scale migration of genome annotations. Nucleic acids research, 43(D1):D690-D697.

Drosophila 12 Genomes Consortium (2007). Evolution of genes and genomes on the Drosophila phylogeny. Nature, 450(7167):203-18. 
Gonzalez, J. and Petrov, D. A. (2012). Evolution of genome content: population dynamics of transposable elements in flies and humans. In Evolutionary Genomics, pages 361-383. Springer.

Hartl, D. L. and Clark, A. G. (1997). Principles of population genetics. Sinauer Associates Sunderland, MA.

Hartl, D. L., Lohe, A. R., and Lozovskaya, E. R. (1997). Modern thoughts on an ancyent marinere: function, evolution, regulation. Annual review of genetics, 31(1):337-358.

Hey, J. (1989). The transposable portion of the genome of drosophila algonquin is very different from that in d. melanogaster. Molecular biology and evolution, 6(1):66-79.

Hickey, D. A. (1982). Selfish DNA: a sexually-transmitted nuclear parasite. Genetics.

Jacobson, J. W., Medhora, M. M., and Hartl, D. L. (1986). Molecular structure of a somatically unstable transposable element in drosophila. Proceedings of the National Academy of Sciences, 83(22):8684-8688.

Kaplan, N., Darden, T., and Langley, C. H. (1985). Evolution and extinction of transposable elements in mendelian populations. Genetics, 109(2):459-480.

Kazazian, H. H. (2004). Mobile elements: drivers of genome evolution. Science, 303:1626-32.

Kazazian Jr, H. H. (1998). Mobile elements and disease. Current opinion in genetics $\&$ development, 8(3):343-350.

Kim, Y. B., Oh, J. H., McIver, L. J., Rashkovetsky, E., Michalak, K., Garner, H. R., Kang, L., Nevo, E., Korol, A. B., and Michalak, P. (2014). Divergence of drosophila melanogaster repeatomes in response to a sharp microclimate contrast in evolution canyon, israel. Proceedings of the National Academy of Sciences, 111(29):10630-10635. 
Kofler, R., Betancourt, A. J., and Schlötterer, C. (2012). Sequencing of pooled dna samples (pool-seq) uncovers complex dynamics of transposable element insertions in Drosophila melanogaster. PLoS genetics, 8(1):e1002487.

Kofler, R., Hill, T., Nolte, V., Betancourt, A., and Schlötterer, C. (2015). The p-element strikes again: the recent invasion of natural drosophila simulans populations. bioRxiv.

Kofler, R., Nolte, V., and Schlötterer, C. (2014). Massive bursts of transposable element activity in Drosophila. bioRXiv.

Kofler, R., Orozco-terWengel, P., De Maio, N., Pandey, R. V., Nolte, V., Futschik, A., Kosiol, C., and Schlötterer, C. (2011). Popoolation: a toolbox for population genetic analysis of next generation sequencing data from pooled individuals. PloS one, 6(1):e15925.

Kurtz, S., Phillippy, A., Delcher, A. L., Smoot, M., Shumway, M., Antonescu, C., and Salzberg, S. L. (2004). Versatile and open software for comparing large genomes. Genome biology, 5(2):R12.

Lachaise, D., Cariou, M. L., David, J. R., and Lemeunier, F. (1988). Historical biogeography of the Drosophila melanogaster species subgroup. Evolutionary Biology, 22:159-222.

Lee, Y. C. G. and Langley, C. H. (2010). Transposable elements in natural populations of Drosophila melanogaster. Philosophical transactions of the Royal Society of London. Series B, Biological sciences, 365(1544):1219-28.

Lerat, E., Burlet, N., Biémont, C., and Vieira, C. (2011). Comparative analysis of transposable elements in the melanogaster subgroup sequenced genomes. Gene, 473(2):100-109.

Li, H. and Durbin, R. (2010). Fast and accurate long-read alignment with Burrows-Wheeler transform. Bioinformatics (Oxford, England), 26(5):589-595.

Lohe, A. R., Moriyama, E. N., Lidholm, D.-A., and Hartl, D. L. (1995). Horizontal transmission, vertical inactivation, and stochastic loss of mariner-like transposable elements. Molecular biology and evolution, 12(1):62-72. 
Loreto, E. L. S., Carareto, C. M. A., and Capy, P. (2008). Revisiting horizontal transfer of transposable elements in Drosophila. Heredity, 100(6):545-54.

MacArthur, R. H. and Wilson, E. O. (1967). The theory of island biogeography, volume 1. Princeton University Press.

Mackay, T., Lyman, R. F., and Jackson, M. S. (1992). Effects of p element insertions on quantitative traits in drosophila melanogaster. Genetics, 130(2):315-332.

Maumus, F., Fiston-Lavier, A.-S., and Quesneville, H. (2015). Impact of transposable elements on insect genomes and biology. Current Opinion in Insect Science.

Meyerson, M., Gabriel, S., and Getz, G. (2010). Advances in understanding cancer genomes through second-generation sequencing. Nature Reviews Genetics, 11(10):685-696.

Montgomery, E. A. and Langley, C. H. (1983). Transposable elements in mendelian populations. ii. distribution of three copia-like elements in a natural population of drosophila melanogaster. Genetics, 104(3):473-483.

Nei, M. and Li, W.-H. (1979). Mathematical model for studying genetic variation in terms of restriction endonucleases. Proceedings of the National Academy of Sciences, 76(10):52695273.

Nolte, V., Pandey, R. V., Kofler, R., and Schlötterer, C. (2012). Genome-wide patterns of natural variation reveal strong selective sweeps and ongoing genomic conflict in Drosophila mauritiana. Genome Research, 23:99-110.

Nunes, M. D. S., Wengel, P. O.-T., Kreissl, M., and Schlötterer, C. (2010). Multiple hybridization events between Drosophila simulans and Drosophila mauritiana are supported by mtDNA introgression. Molecular ecology, 19(21):4695-707.

Nuzhdin, S. V. (1999). Sure facts, speculations, and open questions about the evolution of transposable element copy number. Genetica, 107(1-3):129-137. 
Palmieri, N., Nolte, V., Chen, J., and Schlötterer, C. (2014). Assembly and annotation of Drosophila simulans strains from Madagascar. Genome resources, xx:xx.

Petrov, D. A., Aminetzach, Y. T., Davis, J. C., Bensasson, D., and Hirsh, A. E. (2003). Size matters: non-LTR retrotransposable elements and ectopic recombination in Drosophila. Molecular biology and evolution, 20(6):880-92.

Petrov, D. A., Fiston-Lavier, A.-S., Lipatov, M., Lenkov, K., and González, J. (2011). Population genomics of transposable elements in Drosophila melanogaster. Molecular biology and evolution, 28(5):1633-44.

Quesneville, H., Bergman, C. M., Andrieu, O., Autard, D., Nouaud, D., Ashburner, M., and Anxolabehere, D. (2005). Combined evidence annotation of transposable elements in genome sequences. PLoS computational biology, 1(2):166-175.

R Core Team (2012). R: A Language and Environment for Statistical Computing. R Foundation for Statistical Computing, Vienna, Austria. ISBN 3-900051-07-0.

Sánchez-Gracia, A., Maside, X., and Charlesworth, B. (2005). High rate of horizontal transfer of transposable elements in Drosophila. Trends in genetics : TIG, 21(4):200-3.

SanMiguel, P., Gaut, B. S., Tikhonov, A., Nakajima, Y., and Bennetzen, J. L. (1998). The paleontology of intergene retrotransposons of maize. Nature genetics, 20(1):43-5.

Schaack, S., Gilbert, C., and Feschotte, C. (2010). Promiscuous DNA: horizontal transfer of transposable elements and why it matters for eukaryotic evolution. Trends in ecology $\&$ evolution, 25(9):537-46.

Schlötterer, C., Tobler, R., Kofler, R., and Nolte, V. (2014). Sequencing pools of individuals - mining genome-wide polymorphism data without big funding. Nature Reviews Genetics, 15(11):749-763.

Silva, J. C., Loreto, E. L., and Clark, J. B. (2004). Factors that affect the horizontal transfer of transposable elements. Current issues in molecular biology, 6:57-71. 
Sniegowski, P. D. and Charlesworth, B. (1994). Transposable element numbers in cosmopolitan inversions from a natural population of drosophila melanogaster. Genetics, $137(3): 815-827$.

True, J. R., Mercer, J. M., and Laurie, C. C. (1996). Differences in crossover frequency and distribution among three sibling species of drosophila. Genetics, 142(2):507-523.

Vicoso, B. and Charlesworth, B. (2006). Evolution on the x chromosome: unusual patterns and processes. Nature Reviews Genetics, 7(8):645-653.

\section{Supplementary files}

- Supplementary file 1 Supplementary figures, tables and material and methods (pdf)

- Supplementary file 2 A table containing for every TE family detailed statistics about the number of mapped reads, paired end fragments supporting a TE insertions and TE insertions identified with PoPoolationTE (xlsx)

- Supplementary file 3 A table containing for every TE family the number of lineage specific TE insertions (xlsx)

- Supplementary file 4 A table containing for every TE family the number of low frequency insertions $(f \leq 0.2)(\mathrm{xlsx})$ 